\title{
The Influence of Bound versus Non-Bound Stabilizing Molecules on the Thermal Stability of Gold Nanoparticles
}

Shirin R. King, Susan Shimmon, Daniel D. Totonjian and Andrew M. McDonagh*

School of Mathematical and Physical Sciences, University of Technology Sydney, Broadway, Ultimo, NSW 2007 Australia

* Author to whom correspondence should be addressed; E-Mail: andrew.mcdonagh@uts.edu.au; Tel.: +6129514 1035

\section{Abstract}

Knowledge concerning the sintering behavior of gold nanoparticles (AuNPs) allows for improved nanomaterials for applications such as printed electronics, catalysis and sensing. In this study, we examined the ability of a range of compounds to stabilize AuNPs against thermal sintering and compared compounds with and without functional groups that anchor the molecules to the nanoparticle surface. Thermal stability was characterized in terms of the temperature of the sintering event $\left(T_{\mathrm{SE}}\right)$ as well as thermogravimetric analysis and scanning electron microscopy. We show that anchored stabilizing compounds with high thermal stability are effective at preventing the sintering of AuNPs until the decomposition of the compound. A $T_{\mathrm{SE}}$ of $390{ }^{\circ} \mathrm{C}$ was achieved using 1pyrenebutanethiol as stabilizer. Of the unanchored stabilizers, which were combined with butanethiol-capped AuNPs, two were found to be particularly effective: oleylamine $\left(T_{\mathrm{SE}} \approx 300{ }^{\circ} \mathrm{C}\right)$ and a perylenedicarboximide derivative $\left(T_{\mathrm{SE}} \approx 540{ }^{\circ} \mathrm{C}\right)$, the latter conferring an unprecedented level of thermal stability on ligand-stabilized AuNPs. When selecting stabilizers without anchoring groups, our results demonstrate the importance of choosing those that have an affinity with the capping ligands on the AuNPs to ensure a uniform mixture of AuNPs and stabilizer within a film. 


\section{Introduction}

Gold nanoparticles (AuNPs) continue to attract significant attention due to their unique properties and diverse applications. ${ }^{1-3}$ Their thermal stability is of particular interest as this influences the sintering behavior of ligand-stabilized AuNPs. Generally, low thermal stability is desired where continuous, electrically conducting structures are to be formed at moderate to low temperatures. ${ }^{4-7}$ On the other hand, for applications in sensing and catalysis where the fabrication and operating conditions are often at high temperatures $\left(>300{ }^{\circ} \mathrm{C}\right)$, high thermal stability is necessary to retain the size- and shape-dependent properties of the individual nanoparticles. The most common strategy to induce such high resistance to sintering has been to encapsulate the AuNPs within an inorganic oxide shell ${ }^{8-10}$ or to immobilize the particles on an inorganic oxide substrate. ${ }^{11-16}$

Several factors are known to influence the thermal stability of AuNPs. Longer alkane chain lengths on stabilizing ligands lead to an increased resistance to sintering. ${ }^{6,17,18}$ Particle size has a small effect on the thermal stability, with larger particles sintering at slightly lower temperatures than smaller ones when passivated with the same ligand. This effect has been attributed to the significantly larger volume fraction of passivating ligands on the smaller particles, ${ }^{6}$ although the ligand binding strength has also been shown to vary with particle size, likely due to the differing surface curvatures for different nanoparticle sizes. ${ }^{19}$ Decreasing particle size also considerably reduces the melting temperature of nanoparticles, a phenomenon that affects a wide variety of nanoparticulate materials in addition to gold. ${ }^{10,20}$ The melting point of AuNPs drops rapidly for particles below $5 \mathrm{~nm}$ in diameter, although the melting range of AuNPs as small as $1.5 \mathrm{~nm}\left(\sim 400{ }^{\circ} \mathrm{C}\right)$ is still reasonably high compared to the temperatures where sintering occurs for most alkanethiol-stabilized AuNPs of comparable size $\left(140-220^{\circ} \mathrm{C}\right)^{7,10,21}$

The process of thermally sintering AuNPs is dependent on kinetics and so the rate of heating will affect the temperature of the sintering event $\left(T_{\mathrm{SE}}\right)$ for a particular sample, with slower heating rates 
leading to sintering at lower temperatures. ${ }^{7,22}$ The surrounding atmosphere can also influence the thermal stability of ligand-stabilized AuNPs. Alkanethiol-stabilized AuNPs can be destabilized by oxidation of the sulfur atoms (by atmospheric oxygen), thus weakening the sulfur-gold interaction. ${ }^{22}$ Therefore, heating AuNPs in an inert atmosphere of nitrogen or argon, or under vacuum, prevents oxidation and inhibits sintering until higher temperatures. This suggests that the alkane ligand chain length dependence is due to increased shielding from atmospheric oxygen by longer alkanethiol ligands. ${ }^{7}$ Similarly, the presence of halide-containing surfactants in AuNP samples destabilizes the Au-S bond and lowers the thermal stability when heated under ambient conditions but not when heated under an inert atmosphere. ${ }^{23}$

While it is apparent that the type of stabilizing ligand attached to AuNPs influences their thermal stability, ${ }^{6,19}$ it is unclear if highly stabilizing molecules must be attached to the AuNP surface in order to be effective stabilizers. Recently, we reported the synthesis of a nanocomposite consisting of AuNPs functionalized with a thiolated ruthenium phthalocyanine (RuPc) complex, which showed a surprisingly high thermal stability and resisted sintering up to $\sim 320{ }^{\circ} \mathrm{C} .{ }^{24} \mathrm{The} \mathrm{RuPc}$ complex serves as a robust physical barrier between the AuNPs, preventing sintering until the nanocomposite is heated to the decomposition temperature of the RuPc complex. However, recent work that investigated the stability of the Au-S bond in thiolate-stabilized gold clusters found that the Au-S bond was vulnerable to decomposition at temperatures as low as $150{ }^{\circ} \mathrm{C}$ and that the aryl- or alkylthiolate stabilizers were almost completely removed at $250{ }^{\circ} \mathrm{C} .{ }^{25}$ In the context of thermally stable, ligand-stabilized AuNPs, we therefore hypothesized that the stabilizing ligands need not be anchored to the particles by Au-S bonds, as these will be broken well before the $T_{\mathrm{SE}}$. That is, AuNPs could be stabilized to higher temperatures by the simple inclusion of thermally stable non-thiolated compounds. Increasing the thermal stability by such means may, for example, facilitate studies in probing the optical properties of otherwise thermally sensitive AuNPs at elevated temperatures. 
Herein we report the effects of a range of stabilizing compounds on the thermal stability of AuNPs, in particular comparing compounds with and without functional groups to anchor the molecules to the nanoparticle surface.

\section{Experimental Methods}

General: 1-Pyrenebutanol, 1-butanethiol, 1-octanethiol, 1-dodecanethiol, oleylamine and tetrahydrofuran (Sigma Aldrich), n-octane and 1-hexadecanethiol (Fluka), n-dodecane (Merck) and n-hexadecane (Hopkin \& Williams) were all used as received. Tetrachloroauric acid, ${ }^{26}$ oleylaminecapped AuNPs (OA@AuNPs), ${ }^{27}$ butanethiol-capped AuNPs (BT@AuNPs) ${ }^{28}$ and N,N'-dioleyl3,4,9,10-perylenedicarboximide ${ }^{29}$ were prepared using literature procedures. 1-Pyrenebutanethiol ${ }^{30}$ was prepared using the method of Han and Balakumar ${ }^{31}$ and was purified by column chromatography. $\left[\operatorname{RuPc}\left(4-\mathrm{py}^{t} \mathrm{Bu}\right)_{2}\right]$ was prepared from $\left[\mathrm{RuPc}(\mathrm{PhCN})_{2}\right],{ }^{32}$ using the method of Rawling et al. ${ }^{33}$ Details of these syntheses are described in the Supporting Information. Simultaneous thermogravimetric analysis and differential scanning calorimetry (TGA/DSC) was performed using a TA Instruments SDT Q600. Solid samples weighing $\sim 5 \mathrm{mg}$ and liquid samples weighing $10 \mathrm{mg}$ were loaded into alumina crucibles and heated from room temperature to $700{ }^{\circ} \mathrm{C}$ or higher (up to $1400{ }^{\circ} \mathrm{C}$ ) at $10^{\circ} \mathrm{C} \mathrm{min}^{-1}$ in air at a flow rate of $30 \mathrm{~mL} \mathrm{~min}^{-1}$. Transmission electron microscopy (TEM) was performed using an FEI Tecnai T20 TWIN microscope ( $\left.\mathrm{LaB}_{6}\right)$ operating at $200 \mathrm{keV}$ and fitted with a Gatan 894 2k x 2k camera. Scanning electron microscopy (SEM) was performed using a Zeiss Supra 55VP SEM operating at $20 \mathrm{kV}$, with the samples drop cast onto silicon wafer.

Functionalization of OA@AuNPs with Thiolated Compounds: As-synthesized OA@AuNPs in toluene were washed by adding the colloid dropwise with vigorous swirling to at least 3 times the volume of methanol. The particles were allowed to precipitate and were then collected by centrifugation, rinsed with additional methanol and air-dried. 
To functionalize the OA@AuNPs with alkanethiols, a gold:thiol mole ratio of 1:4 was used. The alkanethiol was dissolved in toluene $(20 \mathrm{~mL})$ and transferred to a single neck round bottom flask equipped with a stirrer bar. The OA@AuNPs were suspended in toluene $(10 \mathrm{~mL})$ and then added to the alkanethiol solution and stirred for 30 min. The flask was left to stand overnight to allow any precipitate to settle. The colloid was collected by pipette, transferred to a clean round bottom flask and the solvent was removed using a rotary evaporator at $45^{\circ} \mathrm{C}$. To remove the displaced oleylamine and excess thiol, ethanol (30 mL) was added to the flask, which was briefly sonicated and the black precipitate was collected by centrifugation. The particles were washed twice more with ethanol and air-dried.

To functionalize OA@AuNPs with 1-pyrenebutanethiol (PyBuSH), excess PyBuSH (8.0 mg) was dissolved in toluene $(20 \mathrm{~mL})$ with sonicating and then transferred to a 2 neck round bottom flask equipped with a stirrer bar. OA@AuNPs $(8.0 \mathrm{mg})$ were suspended in toluene $(10 \mathrm{~mL})$ and then added to the solution of PyBuSH. The solution was stirred under a nitrogen atmosphere for $1 \mathrm{~h}$, and was then transferred to a single neck round bottom flask and the volume of solvent was reduced on a rotary evaporator at $45^{\circ} \mathrm{C}$ until $\sim 3 \mathrm{~mL}$ remained. The concentrated solution was added dropwise to methanol $(30 \mathrm{~mL})$, which was centrifuged to collect the precipitate. The particles were washed twice more with methanol and air-dried.

Preparation of Mixtures at Varying Molecule to Nanoparticle Ratios: Using the density of bulk gold, a $3 \mathrm{~nm}$ spherical AuNP has a mass of $2.73 \times 10^{-19} \mathrm{~g}$. The butanethiol in BT@ AuNPs is $11 \%$ by weight, as measured by TGA (Figure S1), so on average a BT@AuNP has a mass of 3.07 x $10^{-19} \mathrm{~g}$. The mass of a molecule was calculated by dividing its molar mass by Avogadro's number. For a desired ratio of $x$ molecules per nanoparticle, the following formula was used to calculate the masses required:

$$
\mathrm{g}_{\text {stabilizer }} / \mathrm{g}_{\mathrm{BT} @ \text { AuNPs }}=\frac{x}{\mathrm{~m}_{\mathrm{BT} @ A u N P}} \times \mathrm{m}_{\text {stabilizing molecule }}
$$


where $\mathrm{g}_{\text {stabilizer }} / \mathrm{g}_{\mathrm{BT} @ \mathrm{AuNPs}}$ is the ratio of the masses of stabilizer and BT@AuNPs, mBT@AuNP is the average mass in grams of one BT@ AuNP (in this case $3.07 \times 10^{-19} \mathrm{~g}$ ) and $\mathrm{m}_{\text {stabilizing molecule }}$ is the mass in grams of one molecule of stabilizer. The amount of BT@AuNPs in each mixture was $0.5 \mathrm{mg}$, which is an amount found to provide adequate electrical conductivity upon sintering in the absence of additional stabilizing compounds. Stock solutions of materials were prepared and measured volumes were placed into separate vials. Each mixture was then sonicated to thoroughly combine the molecules and AuNPs, and the solvent was evaporated under a gentle stream of compressed air or nitrogen, leaving a dry powder that could be re-dissolved in an appropriate solvent.

Electrical Resistance Measurements: Resistance measurements were performed using a method reported previously. ${ }^{24}$ Briefly, substrates were prepared by coating two gold electrodes onto pieces of thermal oxide-coated silicon wafer. Each sample was dispersed in the minimum amount of chloroform or dry tetrahydrofuran (THF) and then deposited using a fine-tipped glass pipette onto a substrate, such that a continuous film of the sample spanned the two electrodes once the solvent had evaporated. The samples were heated on an aluminium heating block with an attached thermocouple. Steel probes were placed on each electrode and the resistance was measured using a digital multimeter with a maximum reading of $200 \mathrm{M} \Omega$. A LabVIEW program was used to control the heating rate $\left(10^{\circ} \mathrm{C} \mathrm{min}^{-1}\right.$ from room temperature to $\left.450{ }^{\circ} \mathrm{C}\right)$ and to record the temperature and electrical resistance.

SEM Imaging at Different Stages of Sintering: Mixtures were prepared as described above and drop cast from THF onto silicon substrates. One sample was set aside as the unheated sample, and the others were placed on the aluminium heating block used in the setup described above. The samples were heated at $10{ }^{\circ} \mathrm{C} \mathrm{min}-1$ and each one was carefully removed at a certain temperature using tweezers, and then placed on a sheet of steel to cool it to room temperature. Samples were then mounted on SEM stubs without delay and imaged. 


\section{Results \& Discussion}

AuNPs soluble in organic solvents were synthesized using variations of the two-phase BrustSchiffrin method. Using oleylamine (OA) as stabilizing ligand, ${ }^{27}$ 2-6 nm diameter OA@AuNPs were obtained (Figure S2a), where the loosely bound OA could be exchanged for one of a range of thiol compounds. Using 1-butanethiol (BT) as stabilizing ligand, ${ }^{28}$ 2-5 nm diameter BT@AuNPs were synthesized (Figure S2b), which were subsequently mixed with the non-thiolated compounds.

(a)
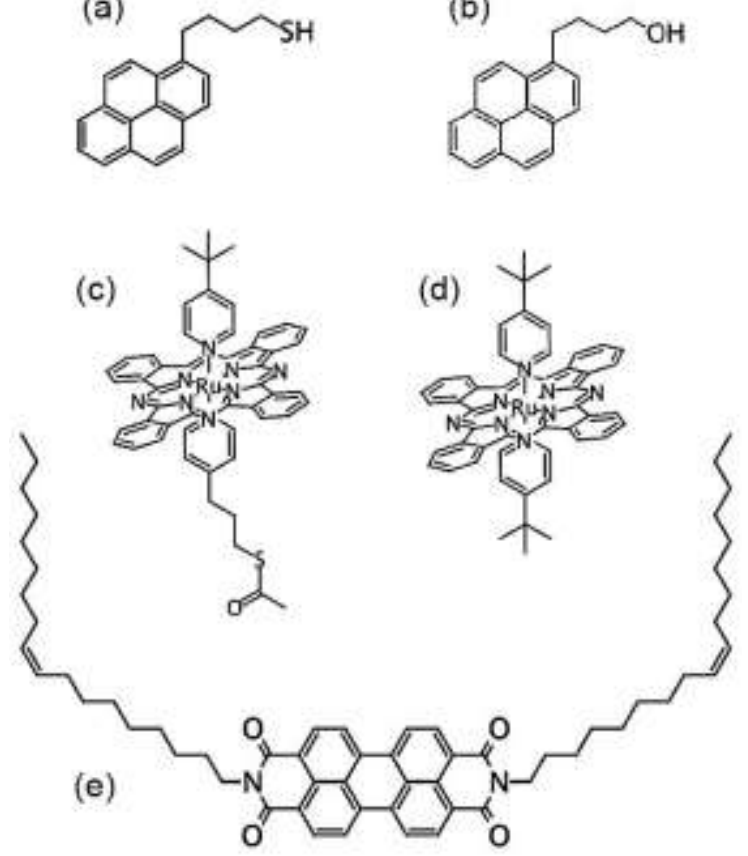

(b)
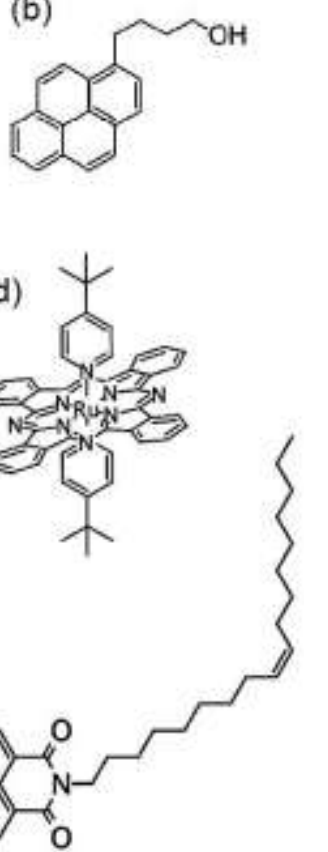

Figure 1. Structures of (a) PyBuSH, (b) $\mathrm{PyBuOH}$, (c) [RuPc(4-py $\left.\left.\left(\mathrm{CH}_{2}\right)_{3} \mathrm{SAc}\right)\left(4-\mathrm{py}{ }^{t} \mathrm{Bu}\right)\right]$, (d) [RuPc$\left.\left(4-\mathrm{py}{ }^{t} \mathrm{Bu}\right)_{2}\right]$ and (e) DPDI. 
Table 1. List of thiolated and analogous non-thiolated stabilizing compounds used in this work.

\begin{tabular}{|c|c|}
\hline Thiol-containing stabilizing ligands & Stabilizers without thiol groups \\
\hline 1-Dodecanethiol (DDT) & Dodecane (DD) \\
\hline 1-Hexadecanethiol (HDT) & Hexadecane (HD) \\
\hline 1-Pyrenebutanethiol (PyBuSH) & 1-Pyrenebutanol (PyBuOH) \\
\hline \multirow{4}{*}{$\begin{array}{l}{[(S \text {-[3-(4-Pyridinyl)propyl] ethanethioate) }(4 \text {-tert- }} \\
\text { butylpyridine)ruthenium phthalocyanine }] \\
\left(\left[\operatorname{RuPc}\left(4-p y\left(\mathrm{CH}_{2}\right)_{3} \mathrm{SAc}\right)\left(4-\mathrm{py}^{t} \mathrm{Bu}\right)\right]\right)^{a}\end{array}$} & [(4-tert-Butylpyridine $)_{2}$ ruthenium phthalocyanine] \\
\hline & $\left(\left[\operatorname{RuPc}\left(4-\mathrm{py}^{\prime} \mathrm{Bu}\right)_{2}\right]\right)$ \\
\hline & Oleylamine (OA) \\
\hline & $\begin{array}{l}N, N^{\prime} \text {-Dioleyl-3,4,9,10-perylenedicarboximide } \\
\text { (DPDI) }\end{array}$ \\
\hline
\end{tabular}

${ }^{a}$ This compound was tested in our previous work. ${ }^{24}$

The stabilizing compounds used in this study are listed in Table 1, and the molecular structures of the less well-known compounds are shown in Figure 1. These compounds were chosen based on their thermal stability (discussed in detail below) and their solubility in the solvents used to dissolve the AuNPs.
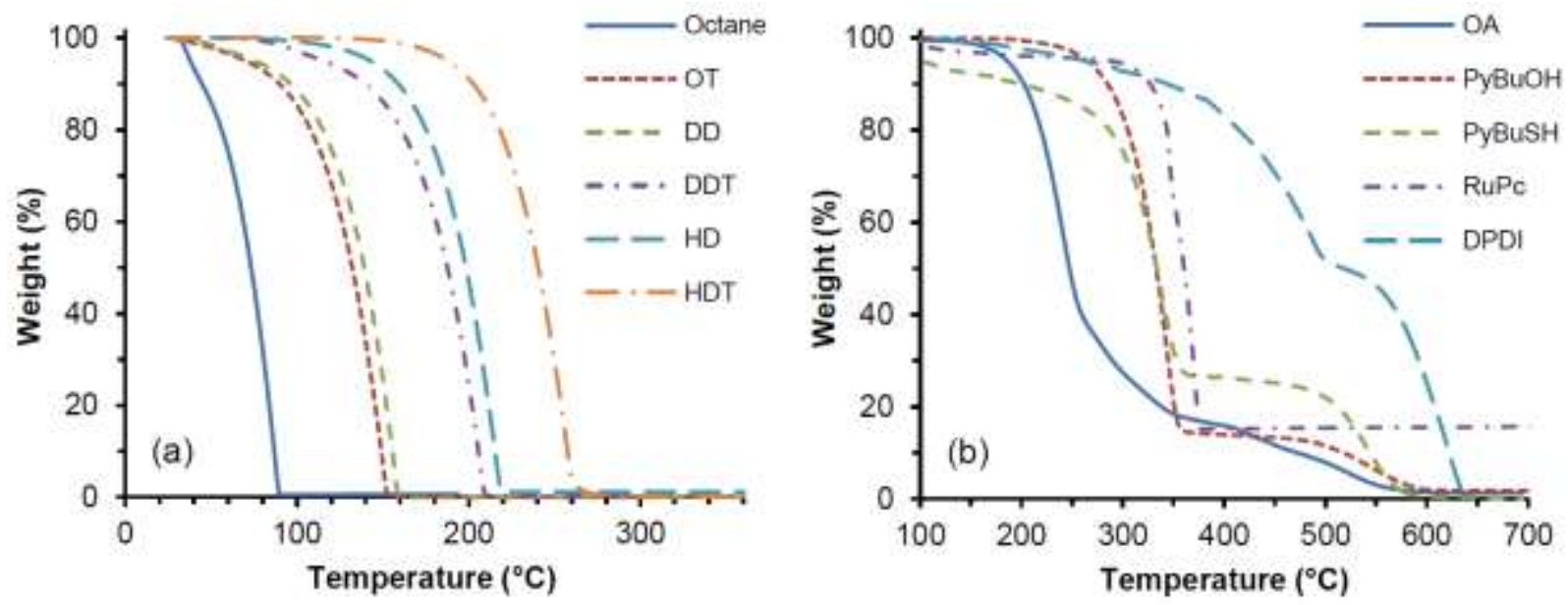

Figure 2. TGA data for (a) the alkanes and alkanethiols and (b) all other stabilizing compounds tested, heated at $10{ }^{\circ} \mathrm{C} \min ^{-1}$ in air. OT $=$ octanethiol, $\operatorname{RuPc}=\left[\operatorname{RuPc}\left(4-\mathrm{py}{ }^{t} \mathrm{Bu}\right)_{2}\right]$. 
The thermal behavior of selected stabilizing compounds was examined using simultaneous TGA/DSC, and the TGA results are shown in Figure 2. All of the alkanes and alkanethiols (Figure 2a) evaporated (evidenced by sharp endotherms in the DSC data, Figure S3) at temperatures 35$75^{\circ} \mathrm{C}$ below their reported boiling points, which we attribute to their volatility and the small volumes used $(\sim 12 \mu \mathrm{L})$. Octane evaporated at a temperature $\sim 70{ }^{\circ} \mathrm{C}$ below the $T_{\mathrm{SE}}$ of BT@ AuNPs (Figure 2 ) and so it was eliminated as a potential stabilizer. The TGA data for the stabilizing compounds shown in Figure 2b reveal two or more mass loss events for each of the compounds, with accompanying exothermic peaks in the DSC data (Figure S4). This indicates that the compounds undergo combustion in stages. Combustion of $\left[\operatorname{RuPc}\left(4-\mathrm{py}{ }^{t} \mathrm{Bu}\right)_{2}\right]$ gave a residue of $\mathrm{RuO}_{2}$ in a process previously described. $^{24}$

The $T_{\mathrm{SE}}$ for each sample of stabilized AuNPs was determined by drop-casting solutions containing the AuNPs (with or without stabilizer) across a pair of gold electrodes on a silicon substrate. The resistance of the specimen was measured whilst heating in air at a constant rate of $10{ }^{\circ} \mathrm{C} \min ^{-1}$. The sintering event is characterized by a significant drop in resistance and we use the definition of $T_{\mathrm{SE}}$ as the temperature of maximum rate of change in resistance. ${ }^{24}$

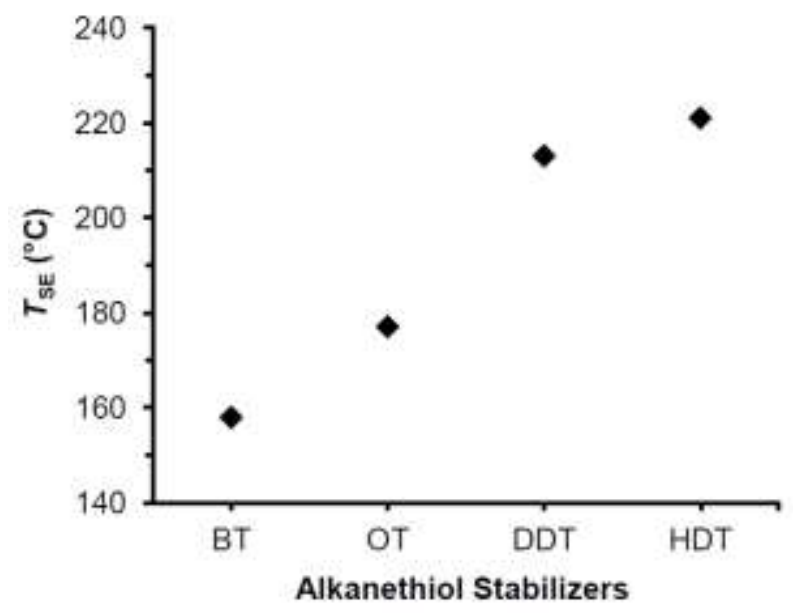

Figure 3. Graph of $T_{\mathrm{SE}}$ data for alkanethiol-stabilized AuNPs. 
Figure 3 shows $T_{\mathrm{SE}}$ data for alkanethiol-stabilized AuNPs with various chain lengths. These data are consistent with previous reports ${ }^{6,17,18}$ and show the effect of increasing $T_{\mathrm{SE}}$ with increasing chain length.

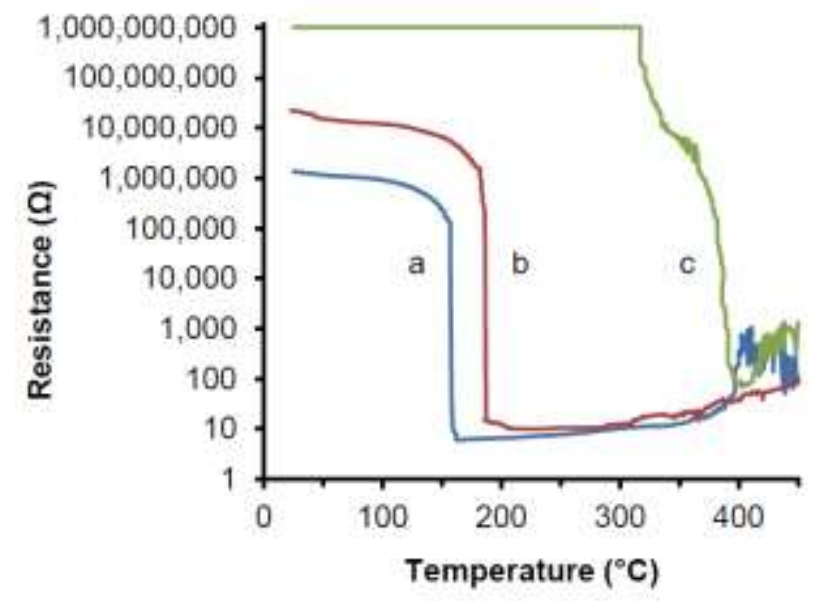

Figure 4. Raw data for measurements of resistance as a function of temperature for (a) BT@AuNPs, (b) BT@AuNPs + PyBuOH at a ratio of 1000 molecules/NP, and (c) PyBuSH@AuNPs, heated at $10{ }^{\circ} \mathrm{C} \mathrm{min}^{-1}$ in air.

Figure 4 shows data obtained during the sintering of BT@AuNPs with and without PyBuOH together with data for AuNPs functionalized with PyBuSH (PyBuSH@AuNPs). The inclusion of PyBuOH raised the $T_{\mathrm{SE}}$ of BT@AuNPs by $\sim 30{ }^{\circ} \mathrm{C}$, however functionalizing AuNPs with PyBuSH resulted in a far higher $T_{\mathrm{SE}}$ of $390^{\circ} \mathrm{C}$, an increase of $\sim 230{ }^{\circ} \mathrm{C}$ compared to BT@AuNPs. 
Table 2. $T_{\mathrm{SE}}$ data for AuNPs with bound and unbound stabilizing compounds. All unbound stabilizers are mixed with BT@AuNPs at a ratio of $\sim 500$ molecules/NP. RuPc $=\left[\operatorname{RuPc}\left(4-p y^{t} \mathrm{Bu}\right)_{2}\right]$ and $\mathrm{RuPcSAc}=[\operatorname{RuPc}(4-$ $\left.\left.\operatorname{py}\left(\mathrm{CH}_{2}\right)_{3} \mathrm{SAc}\right)\left(4-\mathrm{py}^{t} \mathrm{Bu}\right)\right]$.

\begin{tabular}{llll}
\hline Unbound Stabilizer & $\boldsymbol{T}_{\text {SE }}\left({ }^{\circ} \mathbf{C}\right)$ & Bound Stabilizer & $\boldsymbol{T}_{\text {SE }}\left({ }^{\circ} \mathbf{C}\right)$ \\
\hline- & - & BT & 158 \\
DD & 145 & DDT & 213 \\
HD & 154 & HDT & 221 \\
$\mathrm{OA}^{a}$ & 279 & OA $^{a}$ & 323 \\
PyBuOH & 197 & PyBuSH & 390 \\
RuPc & 175 & RuPcSAc & 323 \\
DPDI & 484 & - & - \\
\hline
\end{tabular}

${ }^{a}$ Unbound OA is mixed with BT@AuNPs, while bound OA=OA@AuNPs.

Table 2 shows $T_{\mathrm{SE}}$ data for BT@AuNPs combined with non-thiolated stabilizers together with data for thiol- or OA-stabilized NPs. The BT@AuNPs combined with DD or HD showed little to no increase in thermal stability compared to BT@AuNPs alone. Moderate increases were observed for particles mixed with $\mathrm{PyBuOH}$ or $\left[\mathrm{RuPc}\left(4-\mathrm{py}^{t} \mathrm{Bu}\right)_{2}\right]$. A significant increase in the $T_{\mathrm{SE}}$ of BT@ AuNPs was achieved with the addition of OA. By far the greatest increase in $T_{\mathrm{SE}}$ was achieved upon the addition of DPDI, which can be attributed to its very high thermal stability (Figure $2 \mathrm{~b}$ ). Such a high $T_{\mathrm{SE}}$ is unprecedented for ligand-stabilized AuNPs. 


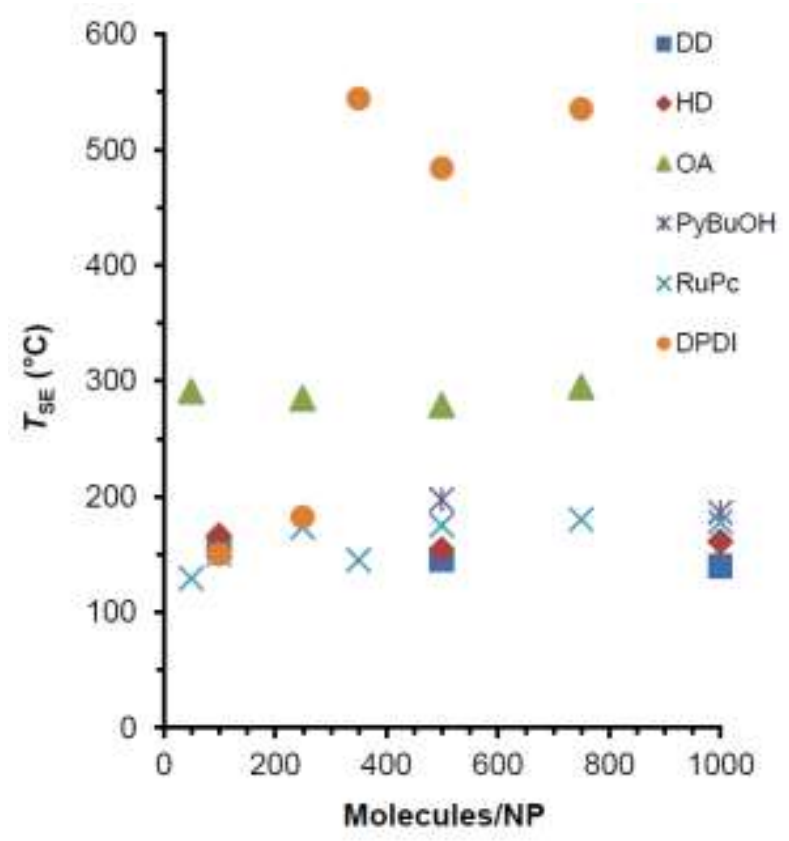

Figure 5. Graph of $T_{\mathrm{SE}}$ data for BT@AuNPs mixed with non-thiolated stabilizers for a range of ratios of molecules/NP. The non-thiolated stabilizers are ordered by thermal stability as determined by TGA. RuPc $=$ $\left[\operatorname{RuPc}\left(4-\mathrm{py}{ }^{t} \mathrm{Bu}\right)_{2}\right]$.

To determine if the $T_{\mathrm{SE}}$ is dependent on the ratio of molecules per NP, particularly since the molecules vary widely in their molecular masses, a range of ratios was tested for each of the nonthiolated stabilizers. For most of the compounds, the $T_{\mathrm{SE}}$ was almost entirely independent of the amount of stabilizer added (Figure 5). For the compound $\mathrm{PyBuOH}$, ratios as high as 2000 and 5000 molecules/NP (data not shown) yielded effectively the same $T_{\mathrm{SE}}$ as with lower ratios. Interestingly, the addition of as little as 50 molecules/NP of OA was found to raise the $T_{\mathrm{SE}}$ of $\mathrm{BT} @ \mathrm{AuNPs}$ to $290{ }^{\circ} \mathrm{C}$, an increase of over $130{ }^{\circ} \mathrm{C}$. It is reasoned that the amine group in OA does not bind to the AuNPs since the particles are already functionalized with BT, which forms a much stronger bond with the gold, although it cannot be discounted that some attraction could take place. DPDI displayed the greatest increase in thermal stability of all the stabilizers tested, with sintering not occurring until $\sim 540{ }^{\circ} \mathrm{C}$ in some samples. This stabilizer also showed some dependence on the ratio of molecules/NP with a threshold ratio of $\sim 300$ molecules/NP, below which the samples sintered at 
temperatures similar to the $T_{\mathrm{SE}}$ of BT@AuNPs. It was observed during experimentation that the samples containing 100 and 250 molecules/NP did not change color from dark red to gold until $\sim 450{ }^{\circ} \mathrm{C}$, despite becoming electrically conductive at 150 and $180{ }^{\circ} \mathrm{C}$, respectively. Generally during sintering, AuNP samples become conductive and change color to lustrous gold simultaneously. It was also observed that use of the waxy DPDI stabilizer resulted in very thick films when deposited on the substrates, particularly in the samples containing $>250$ molecules/NP. Heating these samples led to cracks forming throughout the deposited film, reducing contact between the two electrodes and resulting in a measured final resistance in the low $\mathrm{k} \Omega$ range (Figure S5), in contrast to sintered gold resistances on the order of $10 \Omega$ for the other stabilizers used.

Table 3. $T_{\mathrm{SE}}$ data for OT@AuNPs mixed with HD at different ratios of molecules/NP.

\begin{tabular}{ll}
\hline Molecules/NP & $\boldsymbol{T}_{\mathbf{S E}}\left({ }^{\circ} \mathbf{C}\right)$ \\
\hline 0 & 177 \\
100 & 208 \\
1000 & 208 \\
10000 & 203 \\
\hline
\end{tabular}

The addition of HD had no discernable influence on the $T_{\mathrm{SE}}$ of BT@AuNPs (Figure 5), but adding HD to octanethiol-stabilized AuNPs (OT@AuNPs) increased the $T_{\mathrm{SE}}$ by $\sim 30{ }^{\circ} \mathrm{C}$. The data in Table 3 show that the addition of more than 100 molecules of HD per AuNP did not provide further increases in $T_{\mathrm{SE}}$. The $T_{\mathrm{SE}}$ of OT@AuNPs $+\mathrm{HD}\left(208^{\circ} \mathrm{C}\right)$ is not much less than the $T_{\mathrm{SE}}$ of HDT@AuNPs $\left(221^{\circ} \mathrm{C}\right)$. These data suggest that there are greater intermolecular interactions between HD and the OT in OT@AuNPs than between HD and BT in BT@AuNPs due to the longer carbon chain length of OT compared to BT (Scheme S1). 


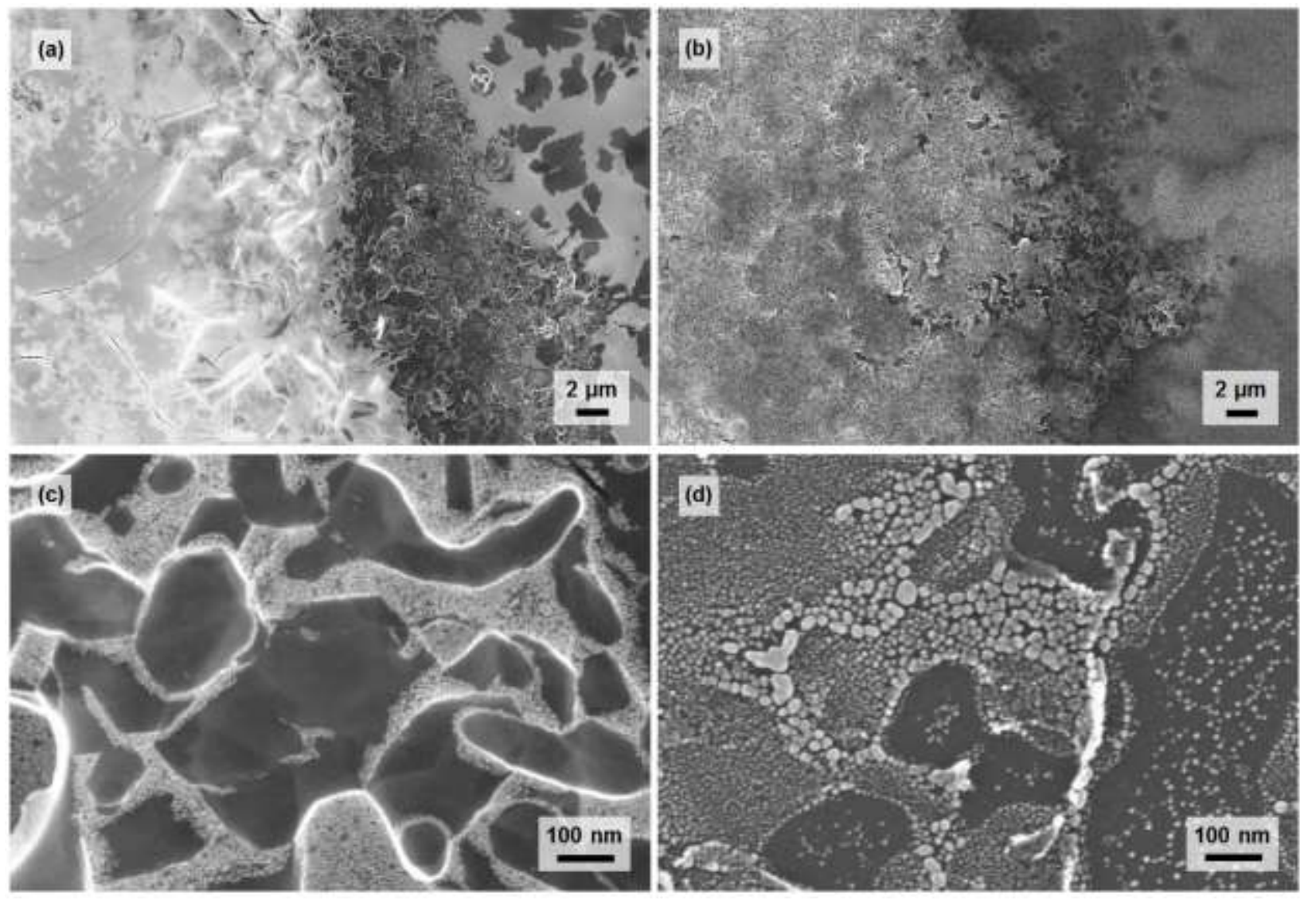

Figure 6. SEM images of $\mathrm{BT} @$ AuNPs mixed with $\left[\mathrm{RuPc}\left(4-\mathrm{py}{ }^{t} \mathrm{Bu}\right)_{2}\right]$ at a ratio of $100 \mathrm{molecules} / \mathrm{NP}$, deposited using chloroform, ( $\mathrm{a}$ and $\mathrm{c}$ ) before heating and (b and d) after heating at $10{ }^{\circ} \mathrm{C} \min ^{-1}$ in air to $350{ }^{\circ} \mathrm{C}$. 
The structures of films of BT@AuNPs mixed with $\left[\mathrm{RuPc}\left(4-\mathrm{py}^{t} \mathrm{Bu}\right)_{2}\right]$ were dependent on the solvent used to deposit the material onto the substrate. Samples deposited from chloroform dried into ring shaped structures (visible to the eye) with most of the material accumulating around the edge of the ring, leaving a thin blue film (from the color of the $\left.\left[\operatorname{RuPc}\left(4-\mathrm{py}^{t} \mathrm{Bu}\right)_{2}\right]\right)$ in the center. These samples sintered at temperatures not much higher than the $T_{\mathrm{SE}}$ of BT@AuNPs (Figure 5), with the edge of the ring changing color from a dark brownish blue to shiny gold while the center remained blue. SEM was conducted to examine the microstructure of these deposited films before and after heating. Figure 6a shows a segment of the ring structure that formed when using chloroform, and Figure $6 \mathrm{~b}$ shows a similar segment after heating, where the AuNPs have sintered. Closer inspection of the inner edge of the ring structure before heating revealed the presence of large clusters of a crystalline material among layers of AuNPs (Figure 6c). These crystals disappeared after heating the sample to $350{ }^{\circ} \mathrm{C}$, leaving an uneven film of sintered AuNPs (Figure 6d). 

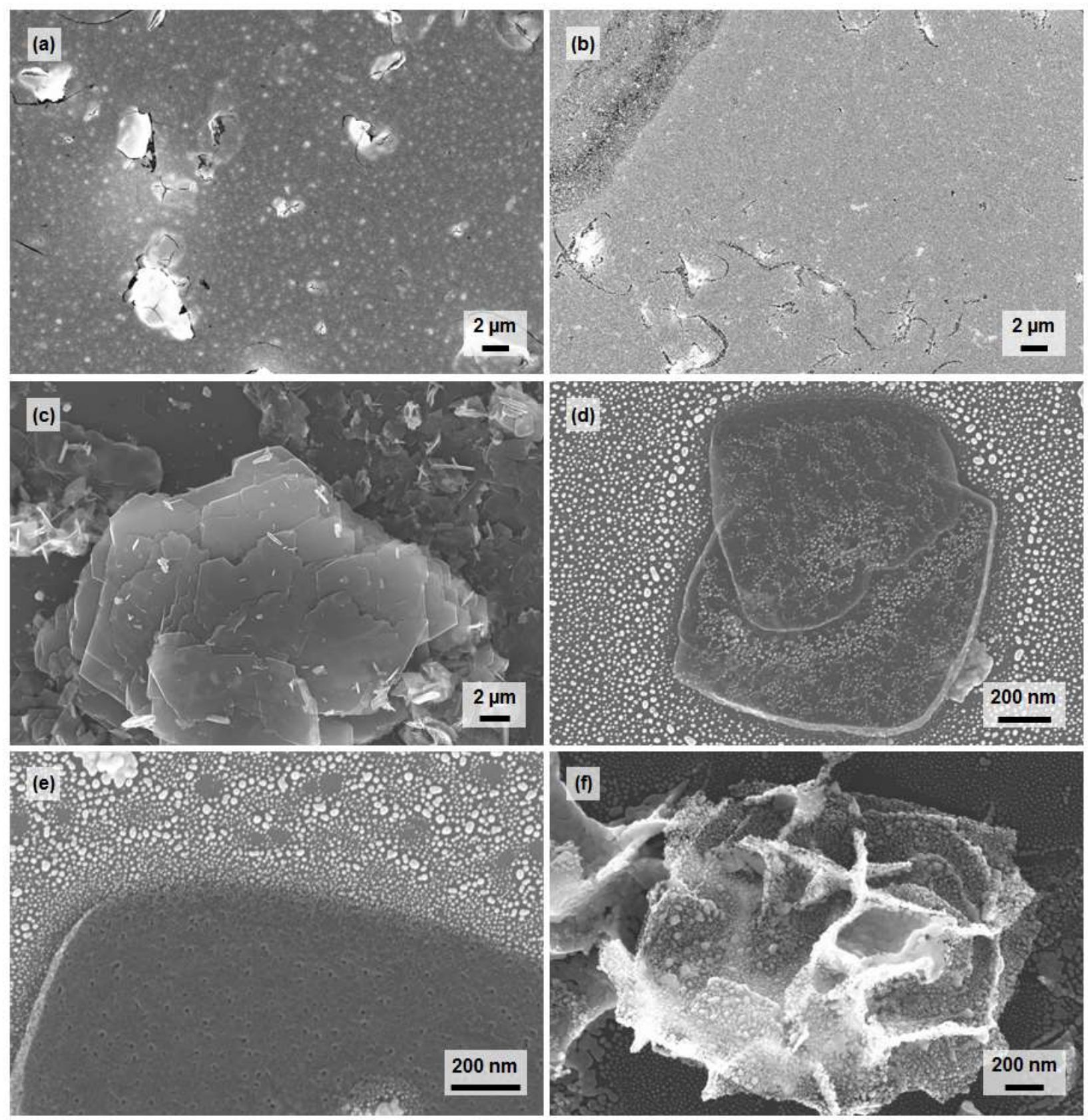

Figure 7. SEM images of mixtures of BT@AuNPs and $\left[\mathrm{RuPc}\left(4-\mathrm{py}^{t} \mathrm{Bu}\right)_{2}\right]$ deposited using THF, at ratios of 100 molecules/NP (a and b) and 250 molecules/NP (c-f). Images (a) and (c) show samples before heating. Images $(\mathrm{d}-\mathrm{f})$ show samples heated at $10{ }^{\circ} \mathrm{C} \mathrm{min}-1$ in air to a temperature of $200{ }^{\circ} \mathrm{C}(\mathrm{d}), 280{ }^{\circ} \mathrm{C}(\mathrm{e})$ and $350{ }^{\circ} \mathrm{C}$ (b and f).

Samples deposited using THF formed far more uniform films after evaporation of the solvent than films deposited using chloroform. Figure 7a shows a reasonably uniform film of unheated AuNPs 
deposited using THF. Clusters of material dotted throughout the sample prior to heating are evident that become less prevalent after heating (Figure 7b). We have identified the clustered material as $\left[\operatorname{RuPc}\left(4-\mathrm{py}^{t} \mathrm{Bu}\right)_{2}\right]$ crystals using SEM images of multiple samples that were prepared with a greater concentration of $\left[\operatorname{RuPc}\left(4-\mathrm{py}^{t} \mathrm{Bu}\right)_{2}\right]$ of 250 molecules/NP and heated to different temperatures. Unheated specimens display a greater abundance of large crystals throughout the sample than in specimens prepared using 100 molecules/NP (Figure S6). Figure 7c shows one of these crystals; greater magnification reveals it to be coated with layers of AuNPs (Figure S7) unlike crystals deposited from chloroform (Figure 6c). At $100{ }^{\circ} \mathrm{C}$ the film of AuNPs in the regions between the crystals (Figure S8) shows the initial sintering stages of diffusion and neck formation between adjacent particles, ${ }^{22,34}$ which by $200{ }^{\circ} \mathrm{C}$ have coarsened into much larger particles of $\sim 20 \mathrm{~nm}$ diameter (Figure 7d), while the AuNPs coated on the crystals show a lesser degree of coarsening at this temperature. After heating to $280{ }^{\circ} \mathrm{C}$, the crystals develop multiple small holes (Figure $7 \mathrm{e}$ ), possibly due to the formation of gaseous combustion products (this temperature is near the onset of decomposition of $\left[\operatorname{RuPc}\left(4-\mathrm{py}^{t} \mathrm{Bu}\right)_{2}\right]$ as measured by TGA (Figure 2)). By $350{ }^{\circ} \mathrm{C}$, the crystals have decomposed leaving behind formations of gold aggregates (Figure 7f) where larger crystals had been, and distinct outlines containing partially sintered AuNPs where smaller crystals had been (Figure S9). These formations persist upon heating to at least $450{ }^{\circ} \mathrm{C}$ (Figure S10).

The film structures observed by SEM for mixtures of BT@AuNPs and [RuPc(4-py $\left.\left.{ }^{t} \mathrm{Bu}\right)_{2}\right]$ demonstrate that without an anchoring group, the stabilizing RuPc compound does not become homogeneously distributed between the AuNPs in the film but instead aggregates into distinct crystals, in contrast to films formed using $\left[\mathrm{RuPc}\left(4-\mathrm{py}\left(\mathrm{CH}_{2}\right)_{3} \mathrm{SAc}\right)\left(4-\mathrm{py}{ }^{t} \mathrm{Bu}\right)\right]$ as stabilizer. ${ }^{24}$ This behavior leaves the AuNPs vulnerable to sintering at lower temperatures than when the stabilizer is anchored directly to the particles. 


\section{Conclusions}

We have investigated the effects of stabilizing compounds with and without anchoring groups on the thermal stability of AuNPs. Most of the unbound stabilizers provided little to no increase in the thermal stability of BT@AuNPs, with the notable exceptions of OA, which gave a $T_{\mathrm{SE}}$ of $\sim 300{ }^{\circ} \mathrm{C}$ even at low concentrations, and DPDI, which at some concentrations gave a $T_{\mathrm{SE}}$ as high as $\sim 540{ }^{\circ} \mathrm{C}$, a result that is unprecedented for ligand-stabilized AuNPs. All of the bound stabilizers conferred greater thermal stability on the AuNPs than their unbound counterparts, with the most effective thiolated stabilizer found to be PyBuSH, giving a $T_{\mathrm{SE}}$ of $390{ }^{\circ} \mathrm{C}$ for PyBuSH@AuNPs. The greater increase in thermal stability observed for mixtures of HD with OT@AuNPs than for HD with BT@AuNPs demonstrates the need for unanchored stabilizing compounds to have an affinity with the capping ligands on AuNPs in order to function as effective stabilizers against sintering. These results, along with the observations made by SEM, suggest the primary role of the anchoring group in a stabilizing compound is to ensure that the molecules are homogeneously distributed between the AuNPs in a film, thereby allowing them to act as physical barriers to sintering until the decomposition of the compound.

\section{Supporting Information}

The supporting information file contains details on the synthesis of stabilizing compounds, TGA/DSC data, TEM images, raw data for selected electrical resistance measurements, a schematic diagram of the interaction between AuNPs and unbound stabilizers, and additional SEM images.

\section{Acknowledgements}

We thank Prof. Michael Cortie, Dr. Angus Gentle, Ms. Melissa Birkett and Mr. Alexander Angeloski for their assistance. This research is supported by an Australian Government Research Training Program Scholarship. 


\section{References}

(1) Daniel, M.-C.; Astruc, D. Gold Nanoparticles: Assembly, Supramolecular Chemistry, Quantum-Size-Related Properties, and Applications toward Biology, Catalysis, and Nanotechnology. Chem. Rev. 2004, 104, 293-346.

(2) Baffou, G.; Quidant, R. Nanoplasmonics for Chemistry. Chem. Soc. Rev. 2014, 43, 38983907.

(3) Eustis, S.; El-Sayed, M. A. Why Gold Nanoparticles Are More Precious than Pretty Gold: Noble Metal Surface Plasmon Resonance and Its Enhancement of the Radiative and Nonradiative Properties of Nanocrystals of Different Shapes. Chem. Soc. Rev. 2006, 35, 209 217.

(4) Wünscher, S.; Abbel, R.; Perelaer, J.; Schubert, U. S. Progress of Alternative Sintering Approaches of Inkjet-Printed Metal Inks and Their Application for Manufacturing of Flexible Electronic Devices. J. Mater. Chem. C 2014, 2, 10232-10261.

(5) Polavarapu, L.; Manga, K. K.; Yu, K.; Ang, P. K.; Cao, H. D.; Balapanuru, J.; Loh, K. P.; Xu, Q.-H. Alkylamine Capped Metal Nanoparticle "Inks" for Printable SERS Substrates, Electronics and Broadband Photodetectors. Nanoscale 2011, 3, 2268-2274.

(6) Huang, D.; Liao, F.; Molesa, S.; Redinger, D.; Subramanian, V. Plastic-Compatible Low Resistance Printable Gold Nanoparticle Conductors for Flexible Electronics. J. Electrochem. Soc. 2003, 150, G412-G417.

(7) Coutts, M. J.; Cortie, M. B.; Ford, M. J.; McDonagh, A. M. Rapid and Controllable Sintering of Gold Nanoparticle Inks at Room Temperature Using a Chemical Agent. J. Phys. Chem. C 2009, 113, 1325-1328.

(8) Lee, I.; Joo, J. B.; Yin, Y.; Zaera, F. A Yolk@Shell Nanoarchitecture for Au/TiO 2 Catalysts. Angew. Chem. 2011, 123, 10390-10393.

(9) Joo, S. H.; Park, J. Y.; Tsung, C.-K.; Yamada, Y.; Yang, P.; Somorjai, G. A. Thermally Stable $\mathrm{Pt} / \mathrm{mesoporous} \mathrm{Silica}$ Core-Shell Nanocatalysts for High-Temperature Reactions. Nat. Mater. 2009, 8, 126-131.

(10) Dick, K.; Dhanasekaran, T.; Zhang, Z.; Meisel, D. Size-Dependent Melting of SilicaEncapsulated Gold Nanoparticles. J. Am. Chem. Soc. 2002, 124, 2312-2317.

(11) Korotcenkov, G.; Cho, B. K.; Gulina, L.; Tolstoy, V. Ozone Sensors Based on $\mathrm{SnO}_{2}$ Films Modified by $\mathrm{SnO}_{2}-\mathrm{Au}$ Nanocomposites Synthesized by the SILD Method. Sensors Actuators B Chem. 2009, 138, 512-517.

(12) Korotcenkov, G.; Brinzari, V.; Cho, B. K. Conductometric Gas Sensors Based on Metal Oxides Modified with Gold Nanoparticles: A Review. Microchim. Acta 2016, 183, 10331054.

(13) Ohodnicki Jr., P. R.; Brown, T. D.; Holcomb, G. R.; Tylczak, J.; Schultz, A. M.; Baltrus, J. P. High Temperature Optical Sensing of Gas and Temperature Using Au-Nanoparticle Incorporated Oxides. Sensors Actuators B Chem. 2014, 202, 489-499.

(14) Stratakis, M.; Garcia, H. Catalysis by Supported Gold Nanoparticles: Beyond Aerobic Oxidative Processes. Chem. Rev. 2012, 112, 4469-4506.

(15) Puértolas, B.; Mayoral, Á.; Arenal, R.; Solsona, B.; Moragues, A.; Murcia-Mascaros, S.; Amorós, P.; Hungría, A. B.; Taylor, S. H.; García, T. High-Temperature Stable Gold 
Nanoparticle Catalysts for Application under Severe Conditions: The Role of $\mathrm{TiO}_{2}$ Nanodomains in Structure and Activity. ACS Catal. 2015, 5, 1078-1086.

(16) Wang, J.; Lu, A.-H.; Li, M.; Zhang, W.; Chen, Y.-S.; Tian, D.-X.; Li, W.-C. Thin Porous Alumina Sheets as Supports for Stabilizing Gold. ACS Nano 2013, 7, 4902-4910.

(17) Martin, J. E.; Odinek, J.; Wilcoxon, J. P.; Anderson, R. A.; Provencio, P. Sintering of Alkanethiol-Capped Gold and Platinum Nanoclusters. J. Phys. Chem. B 2003, 107, 430-434.

(18) Gupta, A.; Mandal, S.; Katiyar, M.; Mohapatra, Y. N. Film Processing Characteristics of Nano Gold Suitable for Conductive Application on Flexible Substrates. Thin Solid Films 2012, 520, 5664-5670.

(19) Smith, B. L.; Hutchison, J. E. Transformations during Sintering of Small ( $\left.D_{\text {core }}<2 \mathrm{~nm}\right)$ Ligand-Stabilized Gold Nanoparticles: Influence of Ligand Functionality and Core Size. $J$. Phys. Chem. C 2013, 117, 25127-25137.

(20) Buffat, P.; Borel, J. P. Size Effect on the Melting Temperature of Gold Particles. Phys. Rev. A 1976, 13, 2287-2298.

(21) Shim, J.-H.; Lee, B.-J.; Cho, Y. W. Thermal Stability of Unsupported Gold Nanoparticle: A Molecular Dynamics Study. Surf. Sci. 2002, 512, 262-268.

(22) Cortie, M. B.; Coutts, M. J.; Ton-That, C.; Dowd, A.; Keast, V. J.; McDonagh, A. M. On the Coalescence of Nanoparticulate Gold Sinter Ink. J. Phys. Chem. C 2013, 117, 11377-11384.

(23) Yu, Y.; Goodfellow, B. W.; Rasch, M. R.; Bosoy, C.; Smilgies, D.-M.; Korgel, B. A. Role of Halides in the Ordered Structure Transitions of Heated Gold Nanocrystal Superlattices. Langmuir 2015, 31, 6924-6932.

(24) King, S. R.; Shimmon, S.; Gentle, A. R.; Westerhausen, M. T.; Dowd, A.; McDonagh, A. M. Remarkable Thermal Stability of Gold Nanoparticles Functionalised with Ruthenium Phthalocyanine Complexes. Nanotechnology 2016, 27, 215702.

(25) Shivhare, A.; Chevrier, D. M.; Purves, R. W.; Scott, R. W. J. Following the Thermal Activation of $\mathrm{Au}_{25}(\mathrm{SR})_{18}$ Clusters for Catalysis by X-Ray Absorption Spectroscopy. J. Phys. Chem. C 2013, 117, 20007-20016.

(26) King, S.; Massicot, J.; McDonagh, A. A Straightforward Route to Tetrachloroauric Acid from Gold Metal and Molecular Chlorine for Nanoparticle Synthesis. Metals. 2015, 5, 1454-1461.

(27) Choi, H.; Chen, W. T.; Kamat, P. V. K. Know Thy Nano Neighbor. Plasmonic versus Electron Charging Effects of Metal Nanoparticles in Dye-Sensitized Solar Cells. ACS Nano 2012, 6, 4418-4427.

(28) Wu, Y.; Li, Y.; Liu, P.; Gardner, S.; Ong, B. S. Studies of Gold Nanoparticles as Precursors to Printed Conductive Features for Thin-Film Transistors. Chem. Mater. 2006, 18, 4627-4632.

(29) Choi, M. J.; Smoother, T.; Martin, A. A.; McDonagh, A. M.; Maynard, P. J.; Lennard, C.; Roux, C. Fluorescent $\mathrm{TiO}_{2}$ Powders Prepared Using a New Perylene Diimide Dye: Applications in Latent Fingermark Detection. Forensic Sci. Int. 2007, 173, 154-160.

(30) Battistini, G.; Cozzi, P. G.; Jalkanen, J. P.; Montalti, M.; Prodi, L.; Zaccheroni, N.; Zerbetto, F. The Erratic Emission of Pyrene on Gold Nanoparticles. ACS Nano 2008, 2, 77-84.

(31) Han, C.-C.; Balakumar, R. Mild and Efficient Methods for the Conversion of Benzylic Bromides to Benzylic Thiols. Tetrahedron Lett. 2006, 47, 8255-8258.

(32) Bossard, G. E.; Abrams, M. J.; Darkes, M. C.; Vollano, J. F.; Brooks, R. C. Convenient 
Synthesis of Water Soluble, Isomerically Pure Ruthenium Phthalocyanine Complexes. Inorg. Chem. 1995, 34, 1524-1527.

(33) Rawling, T.; Austin, C. E.; Hare, D.; Doble, P. A.; Zareie, H. M.; McDonagh, A. M. Thin Films of Ruthenium Phthalocyanine Complexes. Nano Res. 2009, 2, 678-687.

(34) Chen, Y.; Palmer, R. E.; Wilcoxon, J. P. Sintering of Passivated Gold Nanoparticles under the Electron Beam. Langmuir 2006, 22, 2851-2855. 


\section{TOC Graphic}

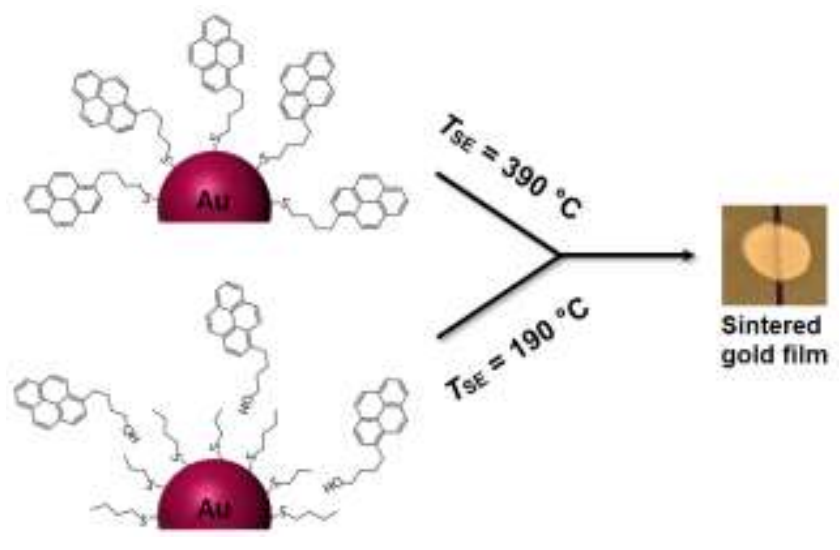

